\title{
LA DIVERSIDAD MORFOLÓGICA in situ DE CHILES SILVESTRES (Capsicum spp.) DE TABASCO, MÉXICO
}

\section{In situ MORPHOLOGICAL DIVERSITY OF WILD PEPPERS (Capsicum spp.) IN TABASCO, MÉXICO}

\author{
Carlos A. Narez-Jiménez ${ }^{1}$, Efraín de-la-Cruz-Lázaro ${ }^{1 \star}$, Armando Gómez-Vázquez ${ }^{1}$, \\ Guillermo Castañón-Nájera ${ }^{2}$, Aldenamar Cruz-Hernández ${ }^{1}$ y César Márquez-Quiroz ${ }^{1}$
}

\footnotetext{
${ }^{1}$ División Académica de Ciencias Agropecuarias, Universidad Juárez Autónoma de Tabasco. Km 25 Carr. Villahermosa-Teapa. Centro, Tabasco, México. ${ }^{2}$ División Académica de Ciencias Biológicas, Universidad Juárez Autónoma de Tabasco. Km 0.5 Carr. Villahermosa-Cárdenas.Villahermosa, Tabasco, México.

*Autor para correspondencia (efrain.delacruz@ujat.mx)
}

\section{RESUMEN}

En el Estado de Tabasco, México se pueden encontrar plantas de chile (Capsicum spp.) de forma silvestre que se diferencian por sus características morfológicas. Con el objetivo de recolectar y evaluar in situ la diversidad morfológica de chiles silvestres, de enero a septiembre de 2013 se realizó la exploración de $\mathbf{4 8}$ comunidades en 14 municipios del Estado de Tabasco. Durante la exploración se recolectaron y tomaron datos de variables de planta, flor y fruto de 89 colectas. Se encontró la presencia de las especies $C$. annuum var. glabriusculum en los morfotipos 'Amashito', 'Ojo de cangrejo' y 'Garbanzo'; y C. frutescens L. en el morfotipo 'Pico paloma'. El análisis de componentes principales y de conglomerados con las 12 variables relevantes, diferenciaron a las colectas en dos grupos, los cuales se formaron con base en variables de fruto y de hoja. El primer grupo se formó con las 43 colectas de C. annuum var. glabriusculum que se caracterizan por tener frutos de forma redonda, corola pequeña de color blanco y una flor por nudo; el segundo grupo se formó con 46 colectas de C. frutescens $\mathrm{L}$. que se caracterizan por tener frutos alargados, corola de color blanca verdosa y de dos a tres flores por nudo. Al interior de los grupos también se presentó una diferencia gradual y continua de las variables, lo que permitió formar subgrupos. En los chiles silvestres del Estado de Tabasco se encuentra diversidad morfológica, recurso genético valioso que debe conservarse.

Palabras clave: Capsicum annuum L., Capsicun annuum var. glabriusculum, Capsicum frutescens L., morfotipos, componentes principales.

\section{SUMMARY}

Wild pepper (Capsicum spp.) plants in the State of Tabasco, México can be differentiated by their morphological characteristics. This research was performed on 89 wild pepper plants collected from 48 communities in $\mathbf{1 4}$ municipalities of Tabasco State from January to September 2013, to evaluate their in situ morphological diversity. Plant, fruit and flower traits were evaluated for each sampled plant. In Capsicum annuum var. glabriusculum species the morphotypes 'Amashito', 'Ojo de cangrejo' and 'Garbanzo' were described, while for $C$. frutescens $\mathrm{L}$. only the 'Pico paloma' morphotype was detected. Principal components and cluster analysis of the 12 selected variables divided the collections into two groups based on fruit and leaf variables. The first group included 43 collections of the C. annuum var. glabriusculum characterized by round-shaped fruits, small white corolla and one flower per axil; the second group comprised 46 collections of the C. frutescens L. characterized by elongated fruits, white greenish corolla and two or three flowers per axil. A gradual and continuous variable differentiation was found within each group which allowed to differentiate subgroups. The morphological diversity in wild pepper plants from Tabasco State is a valuable resource that must be preserved for future uses.

Index words: Capsicum annuum L., Capsicun annuum var. glabriusculum, Capsicum frutescens, morphotypes, principal components.

\section{INTRODUCCIÓN}

El chile (Capsicum spp.) es uno de los cultivos más importantes del mundo. En México existe gran variabilidad de chiles en forma, tamaño, color, sabor y picor, con una distribución desde el nivel del mar hasta los 2500 msnm (Hernández-Verdugo et al., 1999). Junto con el maíz (Zea mays L.), el frijol (Phaseolus vulgaris) y la calabaza (Cucurbita spp.), el chile forma parte de la dieta básica de México desde hace 5000 años; además de ser fuente importante de vitamina $\mathrm{C}$, nuestros antepasados también lo usaron como medicamento, moneda, material de tributo, entre otros (Long, 2011).

El género Capsicum está formado por alrededor de 30 especies, de las cuales solo C. annuum L., C. frutescens $\mathrm{L}$., C. pubescens Ruíz \& Pav., C. chinense Jacq. y C. baccatum L. han sido domesticadas, C. annuum L. es la de mayor importancia económica, ya que se cultiva en todo el mundo y se considera que fue domesticada en México (Hernández, 2011). Además de las cinco especies cultivadas, en estado semidomestícado o silvestre en México se pueden encontrar las especies $C$. annuum L., C. frutescens L., C. ciliatun y C. lanceolatum (Castañón-Nájera et al., 2008).

En el sureste mexicano es posible encontrar poblaciones silvestres de C. annuum L. y C. frutescens L., que presentan variabilidad morfológica y genética (Hernández-Verdugo 
et al., 1999). Tales poblaciones de chiles silvestres no han sido ampliamente estudiadas y ellas representan un recurso en riesgo de extinción, debido a factores adversos como huracanes, sequías y deforestación. Por la necesidad de contar con información sobre la diversidad de chiles silvestres, el objetivo del presente estudio fue recolectar y evaluar in situ la diversidad morfológica de chiles silvestres (C. annuum spp.) del Estado de Tabasco, México.

\section{MATERIALES Y MÉTODOS}

La recolecta y caracterización morfológica in situ de los chiles del Estado de Tabasco se realizó entre enero y septiembre de 2013. El Estado se localiza entre $90^{\circ} 58^{\prime} 08^{\prime \prime}$ y $94^{\circ} 07^{\prime} 00^{\prime \prime} \mathrm{LO}$ y entre $17^{\circ} 15^{\prime} 00^{\prime \prime}$ y $18^{\circ} 38^{\prime} 45^{\prime \prime}$ de LN, sobre la llanura costera del Golfo de México. Tiene una extensión territorial de $24,738 \mathrm{~km}^{2}$, que representa $1.3 \%$ del territorio nacional. El clima predominante es cálido húmedo $A m(f)$ " $(i) g$ con abundantes lluvias en verano, precipitación promedio entre 2500 y $3000 \mathrm{~mm}$, con temperatura media anual de $25{ }^{\circ} \mathrm{C}$ (Ruíz-Álvarez et al., 2012).

Las comunidades de exploración se seleccionaron con base en estudios previos realizados por Castañón-Nájera et al. (2008) y por Pérez-Castañeda et al. (2008), y por información provista por comerciantes de chiles silvestres de mercados de los municipios explorados. Se obtuvieron 89 colectas en los recorridos de caminos, huertos, potreros y ecosistemas de plátano y cacao, en 48 comunidades de 14 municipios del Estado. En cada lugar de colecta se registraron las coordenadas con GPS Garmin eTrex ${ }^{\circledR}$ las cuales se marcaron en el mapa (Figura 1).

Todos los frutos maduros de la planta o plantas muestreadas, se guardaron en bolsas de papel y se trasladaron al Centro de Investigación en Ciencias Agropecuarias de la Universidad Juárez Autónoma de Tabasco, donde se dejaron secar a temperatura ambiente para después extraer la semilla y obtener el número de semilla por fruto.

En las plantas muestreadas se evaluaron 24 variables morfológicas de planta, flor y fruto, de acuerdo con los descriptores para Capsicum del IPGRI, CATIE, AVRDC (1995). Tales descriptores fueron, para planta: altura de planta, ancho de planta, diámetro de tallo, pubescencia de tallo, hábito de crecimiento, densidad de ramificación, color de hoja, forma de hoja, margen de la lámina foliar, pubescencia de hoja, longitud de hoja, ancho de hoja; en flor: número de flores por nudo, posición de la flor, color de corola y forma de corola; y en fruto: color de fruto, forma del fruto, forma del fruto en la unión con el pedicelo, forma del ápice del fruto, número de semillas por fruto, largo de fruto, ancho de fruto y peso de fruto. Se evaluaron 89 colectas de chiles silvestres de los morfotipos: 'Pico paloma' (46), 'Garbanzo'
(28), 'Amashito' (11) y 'Ojo de cangrejo' (4). Con base en variables de fruto y flor se identificó la especie a la que pertenecen las colectas (Andrews, 1995; Hernández-Verdugo et al., 1999; Bosland y Votava, 2012).

Con las medias y las modas de cada variable por colecta se realizó un primer análisis de componentes principales (ACP) que incluyó 89 colectas y 24 variables, las cuales se estandarizaron a $\mu=0$ y $\sigma^{2}=1$. Este análisis permitió determinar las 12 variables de mayor importancia para describir la variabilidad de las colectas, con las cuales se procedió a realizar un segundo ACP. La significancia de los valores y vectores "eigen” se determinó con la regla de Keiser (1960). En ambos análisis, la estimación de los CP se hizo con la matriz de correlaciones, con la finalidad de que las variables involucradas en el análisis tuvieran la misma importancia.

Después de seleccionar las variables de mayor valor descriptivo, se procedió a hacer un análisis de conglomerados jerárquicos con el método de ligamiento promedio (UPGMA), con la distancia euclidiana como media de disimilitud, y con la matriz de distancias se construyó el dendrograma. El punto de corte para la formación de grupos se basó en la $R^{2}$ semiparcial mayor a $70 \%$ y en las pruebas de la seudo F y la seudo $t^{2}$ donde las diferencias entre grupos fueron significativas (SAS, 2009).

\section{RESULTADOS Y DISCUSIÓN}

El primer análisis de componentes principales (ACP) con las 24 variables mostró que con los tres primeros componentes principales (CP) se explica $46.93 \%$ de la variabilidad morfológica total. Al respecto, se sabe que al eliminar las variables que aportan poco o nada a la explicación del CP1, se mejora en forma general el ACP (Castañón-Nájera et al., 2008). Al hacer el ACP con las 12 variables seleccionadas, los tres primeros CP explicaron $73.27 \%$ de la variabilidad total (Cuadro 1), y los primeros tres CP presentaron valores significativos de acuerdo con el criterio de Keiser, (1960) .

El CP1 explicó 43.61 \% de la variación total, y las variables de mayor contribución fueron: ancho de fruto, forma de fruto, forma del fruto en la unión con el pedicelo, largo de fruto, forma de ápice del fruto y color de fruto. Es decir este componente estuvo conformado por variables de fruto, lo cual es consistente con estudios previos sobre diversidad morfológica de chiles que reportan que el primer componente fue explicado por variables de fruto (Castañón-Nájera et al., 2008). El CP2 explicó el 18.50 \% de la variación total, siendo las variables largo de hoja, ancho de hoja, color de hoja y número de semillas por fruto las que en mayor proporción influyeron en la determinación del componente. De la misma forma, el CP3 contribuyó con el 11.16 \% de la variación y se relacionan de forma positiva con las variables hábito de 


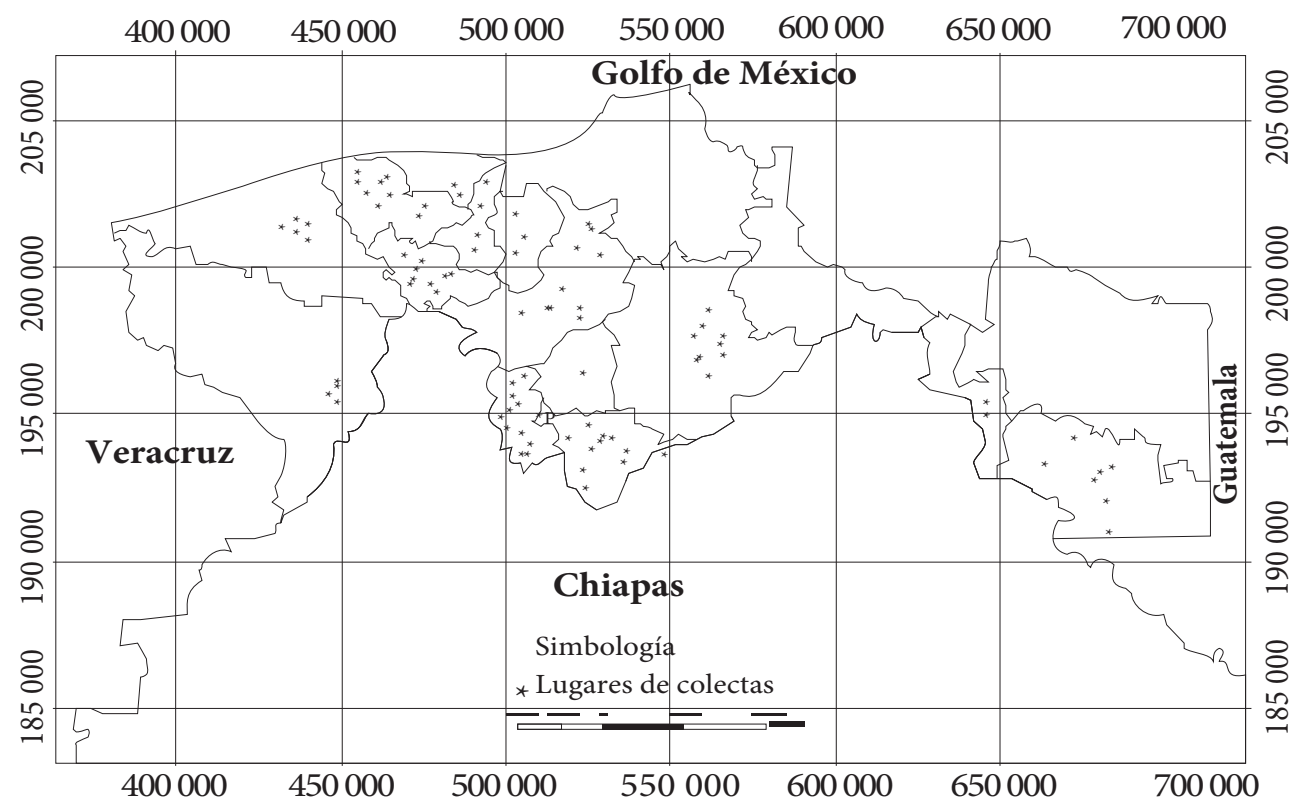

Figura 1. Localización geográfica de los lugares donde se hicieron las 89 colectas silvestres de C. annuum var. glabriusculum y $C$. frutescens $\mathrm{L}$. en el Estado de Tabasco.

Cuadro 1. Vectores y valores de los componentes principales (CP) con las variables de planta, flor y fruto de mayor valor descriptivo de 89 chiles (Capsicum spp.) silvestres del Estado de Tabasco.

\begin{tabular}{llll}
\hline Variable & CP1 & CP2 & CP3 \\
\hline Diámetro de tallo & 0.1142 & 0.1107 & $0.2625^{\dagger}$ \\
Hábito de crecimiento & 0.1492 & -0.0044 & $0.5224^{\dagger}$ \\
Color de hoja & 0.0326 & $0.3646^{\dagger}$ & $-0.4571^{\dagger}$ \\
Largo de hoja & 0.0301 & $0.6154^{\dagger}$ & -0.1057 \\
Ancho de hoja & 0.0446 & $0.5813^{\dagger}$ & -0.0650 \\
Largo de fruto & $0.4218^{\dagger}$ & -0.0028 & -0.0129 \\
Ancho de fruto & $0.4301^{\dagger}$ & 0.0033 & -0.0295 \\
Forma del fruto & $0.4238^{\dagger}$ & -0.0930 & -0.1175 \\
Forma del fruto en la unión con el pedicelo & $0.4230^{\dagger}$ & -0.0830 & -0.1175 \\
Forma del ápice del fruto & $0.4139^{\dagger}$ & -0.0891 & -0.1292 \\
Color de fruto & $0.2564^{\dagger}$ & 0.2219 & $0.4005^{\dagger}$ \\
Número de semillas por fruto & -0.0387 & $0.2523^{\dagger}$ & $0.4777^{\dagger}$ \\
Valor propio & $5.2336^{\dagger}$ & $2.2199^{\dagger}$ & $1.3393^{\dagger}$ \\
Variación explicada & 0.4361 & 0.1850 & 0.1116 \\
Variación acumulada & 0.4361 & 0.6211 & 0.7327 \\
\hline
\end{tabular}

${ }^{\dagger}$ Significativo, de acuerdo con Keiser (1960). 
crecimiento, número de semillas por fruto, color de fruto y diámetro de tallo.

La distribución de los morfotipos de chiles mediante los componentes principales $\mathrm{CP} 1$ y CP2 (Figura 2) dio lugar a dos grupos, el primer con los morfotipos 'Amashito', 'Ojo de cangrejo' y 'Garbanzo', mientras que el segundo grupo se formó con los chiles del morfotipo 'Pico paloma'. Las colectas del primer grupo se ubicaron en el cuadrante positivo del CP1 y en los cuadrantes positivo y negativo del CP2; en este grupo se encuentran colectas con los frutos de menor peso, de forma redonda y frutos con ápice romo o hundido. Las colectas del segundo grupo se ubicaron en el cuadrante negativo del $\mathrm{CP} 1$ y se distribuyeron en los cuadrantes positivo y negativo del CP2, e incluye a las colectas con los frutos de mayor peso, de forma alargada y ápice del fruto puntiagudo.

Los morfotipos del primer grupo presentan características de la especie C. annuum var. glabriusculum, debido a que presentan frutos pequeños de forma redonda, deciduos, erectos, con una flor pequeña de color blanca por nudo (Andrews, 1995 y Hernández-Verdugo et al. 1999). Al respecto, Castañón-Nájera et al. (2008) reportan que los morfotipos 'Garbanzo' y 'Amashito' pertenecen a esta especie; y Pérez-Castañeda et al. (2008) reportaron que el morfotipo 'Ojo de cangrejo' pertenece a Capsicum annuum. Las colectas del morfotipo 'Pico paloma' presentaron frutos alargados puntiagudos, con dos o más flores por nudo de color blanca verdosa, características que según Andrews (1995), Hernández-Verdugo et al. (1999) y Bosland y Votava (2012) presenta la especie $C$. frutescens $\mathrm{L}$. Similarmente, Hernández-Verdugo et al. (1999) reportaron que en el Sureste de México se pueden encontrar poblaciones silvestres de C. annuum var. glabriusculum y C. frutescens $\mathrm{L}$. con gran variabilidad morfológica y genética. También CastañónNájera et al. (2008) y Pérez-Castañeda et al. (2008) reportaron la presencia de estas dos especies de chiles silvestres en el Estado de Tabasco.

Con en el análisis de conglomerados fue posible agrupar a las 89 colectas en dos grupos morfológicamente diferentes (Figura 3), de acuerdo con el $R^{2}$ semiparcial mayor a 70 $\%$, con la seudo F y la seudo $t^{2}$ significativas (SAS, 2009), al tomar como referencia una distancia euclidiana de 1.18 unidades. El grupo 1 estuvo integrado por las colectas de los morfotipos 'Amashito', 'Garbanzo' y 'Ojo de Cangrejo', las que por sus características morfológicas de flor y fruto pertenecen a la especie $C$. annuum var. glabriusculum, que es la forma silvestre del chile cultivado (Hernández-Verdugo et al., 2012). Este grupo se caracteriza por tener plantas con altura promedio de $116 \mathrm{~cm}$, peso de fruto de $0.32 \mathrm{~g}$, largo de fruto de $8.8 \mathrm{~mm}$, ancho de $6.8 \mathrm{~mm}$ y 15 semillas por fruto, valores que están dentro de los reportados para la especie por otros investigadores (Hernández-Verdugo et al., 1999; Pérez-Castañeda et al., 2008).

Dentro del Grupo 1 se formaron dos subgrupos a una distancia de 0.75 unidades, el primero (IA) incluyó las colectas de los morfotipos 'Amashito', 'Ojo de cangrejo' y 15 colectas de 'Garbanzo', que tuvieron los menores pesos de fruto. En general, este grupo se formó por colectas de frutos pequeños $(7.9 \mathrm{~mm}$ de largo, peso de $0.20 \mathrm{~g}$ y 12.3 semillas por fruto), hojas pequeñas ( $35 \mathrm{~mm}$ de largo y 20 $\mathrm{mm}$ de ancho) y altura de planta de $105 \mathrm{~cm}$. El subgrupo IB se formó por 13 colectas del morfotipo 'Garbanzo' que se caracterizan por tener los mayores pesos y largo de fruto $(0.6 \mathrm{~g}$ y $12 \mathrm{~mm})$, altura de planta $(140 \mathrm{~cm})$ y tamaño de hoja (46 $\mathrm{mm}$ de largo y $27 \mathrm{~mm}$ de ancho).

Al respecto, Hernández-Verdugo et al. (2012) reportaron que el peso de fruto se relaciona con la precipitación y la temperatura media anual durante el desarrollo de la planta, por lo que el mayor peso de fruto de estas colectas se puede deber a que provienen de municipios localizados en la subregión Sierra de Tabasco donde se registra alta precipitación y la menor temperatura promedio anual del Estado (Ruíz-Álvarez et al., 2012). En general, el largo (15 mm) y ancho $(10 \mathrm{~mm})$ de fruto de las colectas de este grupo no superó los valores máximos reportados para la especie C. annuum var. glabriusculum (Hernández-Verdugo et al., 1999).

El Grupo 2 estuvo formado por las 46 colectas del morfotipo 'Pico paloma' que pertenecen a $C$. frutescens $\mathrm{L}$., cuyos frutos tuvieron un peso promedio de $0.70 \mathrm{~g}$, largo de $17 \mathrm{~mm}$ y ancho de $5.3 \mathrm{~mm}, 19$ semillas por fruto y altura de planta de $126 \mathrm{~cm}$; estos valores se ubican dentro de los tamaños reportados para frutos silvestres de C. frutescens $\mathrm{L}$. (Castañón-Nájera et al., 2008; Pérez-Castañeda et al., 2008). En este Grupo 2 se distinguen dos subgrupos a una distancia de 0.75 unidades ambos formados por 23 colectas cada uno.

El Subgrupo IIA se formó con 23 colectas que tuvieron los menores tamaños y pesos de fruto ( $16 \mathrm{~mm}$ de largo, $5.1 \mathrm{~mm}$ de ancho, peso de $0.57 \mathrm{~g}$ y 13.5 semillas por fruto), hojas pequeñas (33.6 $\mathrm{mm}$ de largo y $19.5 \mathrm{~mm}$ de ancho) y altura de planta de $121 \mathrm{~cm}$. El Subgrupo IIB se formó por 23 colectas que se caracterizan por tener las plantas con los frutos de mayor tamaño: $18 \mathrm{~mm}$ de largo, $6.0 \mathrm{~mm}$ de ancho, $0.90 \mathrm{~g}$ de peso y 21 semillas por fruto, plantas de $130 \mathrm{~cm}$ con hojas de $41.7 \mathrm{~mm}$ de largo y $22.7 \mathrm{~cm}$ de ancho. Dentro de las colectas de este subgrupo sobresale la colecta 141P con el mayor tamaño de fruto, largo de $40.8 \mathrm{~mm}$, ancho de $6.9 \mathrm{~mm}$ y peso de $1.20 \mathrm{~g}$. En general las 46 colectas del Grupo 2 se encuentran dentro del rango reportado de longitudes y pesos para C. frutescens $L$. (Bosland y Votava, 2012). 


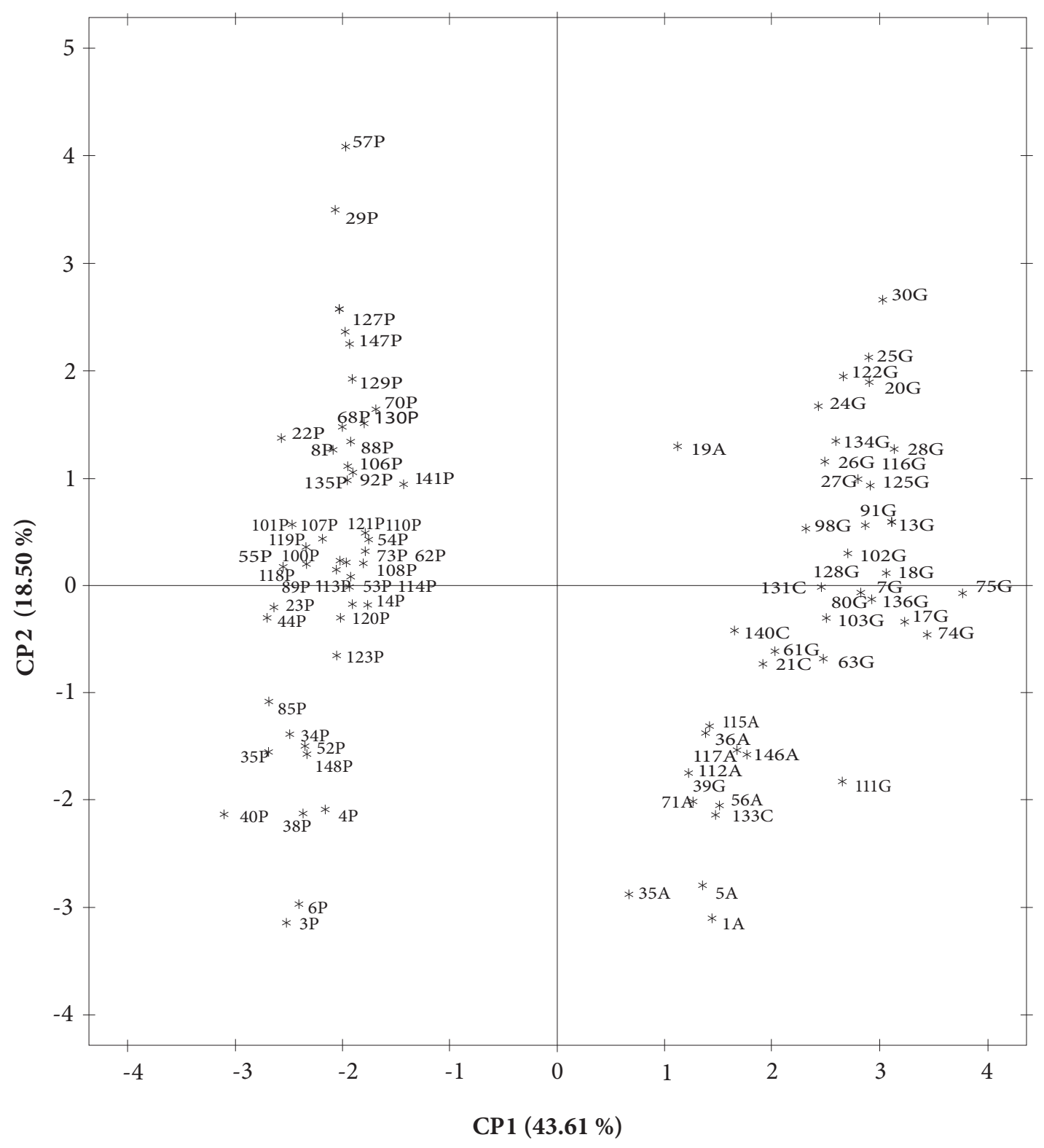

Figura 2. Distribución gráfica de las 89 colectas de C. annuum var. glabriusculum y C. frutescens L. en función de los primeros dos componentes principales.

Las especies C. annuum var. glabriusculum y C. frutescens L. están presentes en todos los municipios incluidos en el estudio, con predominio de los morfotipos 'Pico paloma' (51.69 \%), 'Garbanzo' (31.46 \%) y en menor proporción 'Amashito' (12.36 \%) y 'Ojo de cangrejo' (4.49\%). La menor proporción de los morfotipos 'Ojo de Cangrejo' y 'Amashito', se debe a que son los morfotipos preferidos para consumo y venta local para acompañar platillos típicos de la gastronomía del Estado (Pérez-Castañeda et al., 2008).

La variabilidad morfológica encontrada en las 89 muestras de chiles silvestres colectadas en el Estado de Tabasco, correspondientes a las especies C. annuum var. glabriusculum ('Amashito', 'Ojo de cangrejo' y 'Garbanzo') y C. frutescens L. ('Pico paloma'), indica que estos chiles constituyen un recurso genético valioso que amerita ser estudiado para mejorar su uso y conservación, y como reservorio de genes con potencial para solucionar problemas agrícolas.

\section{AGRADECIMIENTOS}

Al Programa de Fomento a la Investigación por el apoyo al proyecto con clave UJAT-2013-IB-23. 


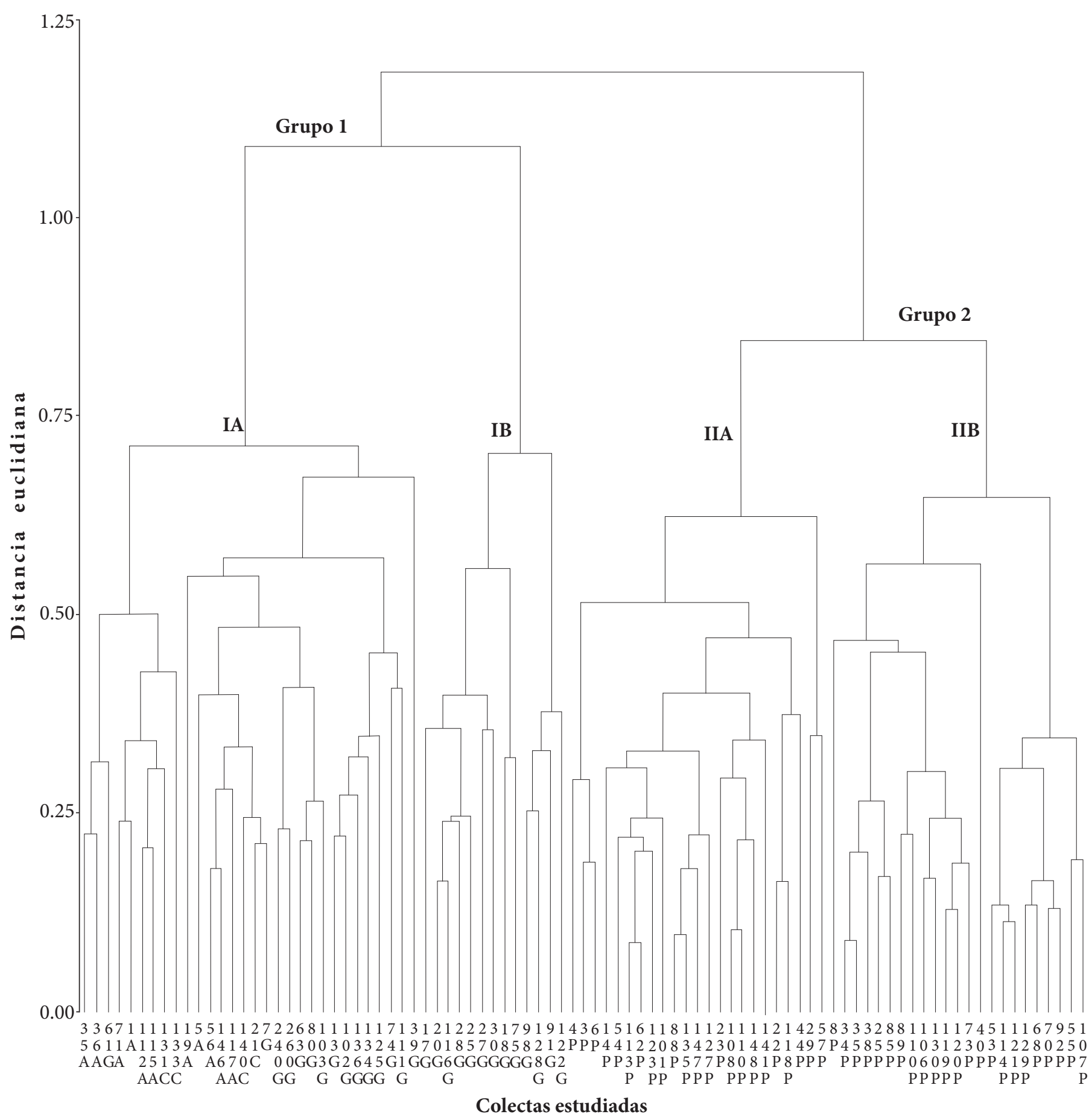

Figura 3. Agrupamiento por el método UPGMA de 89 colectas de chiles (C. annuum var. glabriusculum y C. frutescens L.) donde A: 'Amashito', C: 'Ojo de cangrejo', G: 'Garbanzo' y P: 'Pico paloma'. 


\section{BIBLIOGRAFÍA}

Andrews J. (1995) Peppers: The domesticated Capsicum. New edition. University of Texas Press, Austin, Texas. 186 p.

Bosland P. W. and E. J. Votava (2012) Peppers: vegetable and spice Capsicums. 2nd (Ed.). Cabi publishing. London, UK. 230 p.

Castañón-Nájera G., L. Latournerie-Moreno, M. Mendoza-Elos, A. Vargas-López y H. Cárdenas-Morales (2008) Colección y caracterización de chile (Capsicum spp.) en Tabasco, México. Phyton- Revista Internacional de Botánica Experimental 77:189-202.

Hernández V. S. (2011) Los parientes silvestres del chile: su importancia biológica y cultural. In: El Chile. Protagonista de la independencia y la revolución. K. Richterich (Coord. Edit.). Fundación Herdez. México, D.F. pp:21-26.

Hernández-Verdugo S., F. Porras, A. Pacheco-Olvera, R. G. LópezEspaña, M. Villarreal-Romero, S. Parra-Terraza y T. Osuna E. (2012) Caracterización y variación ecogeográfica de poblaciones de chiles (Capsicum annuum var. glabriusculum) silvestre del Noroeste de México. Polibotánica 33:175-191.

Hernández-Verdugo S., R. G. Guevara-González, R. F. Rivera-Bustamante, C. Vázquez-Yanes y K. Oyama (1999) Los parientes silvestres del chile (Capsicum spp.) como recursos genéticos Boletín de la Sociedad Botánica de México 62:171-181.

IPGRI, AVRDC y CATIE (1995) Descriptores para Capsicum (Capsicum spp.) Instituto Internacional de Recursos Genéticos (IPGRI) Italia; Centro Asiático para el Desarrollo y la Investigación Relativos a los Vegetales (AVRDC), Taipei, Taiwan; y Centro Agronómico Tropical de Investigación y Enseñanza (CATIE), Turrialba, Costa Rica. $51 \mathrm{p}$

Keiser H. F. (1960) The application of electronic computers to factor analysis. Educational and Phsychological Measurement 20:141-151.

Long T. J. (2011) El Capsicum a través de la historia Mexicana. In: El Chile. Protagonista de la independencia y la revolución. K. Richterich (Coord. Edit.) Fundación Herdez. México D.F. pp:7-19.

Pérez-Castañeda L. M., G. Castañón-Nájera y N. Mayek-Pérez (2008) Diversidad morfológica de chiles (Capsicum spp.) de Tabasco, México. Cuadernos de Biodiversidad 27:11-22.

Ruíz-Álvarez O., R. Ortega-Ramírez, M. A. Vázquez-Peña, R. E. Ontiveros C. y R. López-López (2012) Balance hídrico y clasificación climática del estado de Tabasco, México. Universidad y Ciencia 28:1-14.

SAS Institute (2009) SAS/STAT® 9.2 User's Guide. SAS Inst. Inc., Cary, NC, USA. 